\title{
ESTIMATIVA DE BIOMASSA DA FLORESTA OMBRÓFILA DENSA DE TERRA FIRME NA AMAZÔNIA CENTRAL COM O SATÉLITE IKONOS II
}

\author{
Francisca Dionízia de Almeida Matos*, Flávio Felipe Kirchner** \\ ${ }^{*}$ Eng ${ }^{\text {a }}$. Florestal, Dra ${ }^{\mathrm{a}}$, Instituto Nacional de Pesquisas da Amazônia, INPA - fmatosam@gmail.com \\ ${ }^{* *}$ Eng. Florestal, Ph.D., Depto. de Ciências Florestais, UFPR - kirchner@ufpr.br \\ Recebido para publicação: 22/05/2006 - Aceito para publicação: 01/10/2007
}

\begin{abstract}
Resumo
A implicação da redução das florestas tropicais no ciclo de carbono vem criando uma demanda de desenvolvimento de métodos não-destrutivos para a determinação de biomassa, elemento importante na modelagem dos ciclos biogeoquímicos. A pesquisa teve por objetivo estimar indiretamente a biomassa acima do solo de uma floresta primária, correlacionando dados de campo e orbitais do satélite IKONOS II. A biomassa foi obtida pelo método indireto e a análise da vegetação por meio da estrutura horizontal. Na modelagem foram usadas as bandas originais, Índices de Vegetação, como ARVI, NDVI, MSAVI, R43, e Transformações por Componentes Principais, Tasseled Cap e Modelo Linear de Mistura Espectral. A integração da verdade de campo e os dados de satélite foi realizada por procedimentos estatísticos, aplicando-se regressão linear múltipla usando o método Stepwise. Para a espacialização, adotou-se como classes os valores mínimos e máximos dos limites de confiança das estimativas de biomassa obtidas com o modelo. O intervalo de confiança a $95 \%$ de probabilidade para a biomassa encontrada no campo foi de 343,05 $\pm 14,90 \mathrm{Mg}_{\text {.ha }}{ }^{-1}$. As maiores correlações entre biomassa e as variáveis digitais ARVI, MLME $E_{\mathrm{VEG}}$ e NDVI são negativas. A modelagem mostrou que as variáveis R43, NDVI, TCap3 e TCap4 foram as que melhor se correlacionaram com a biomassa.

Palavras-chave: Biomassa acima do solo; modelagem; sensoriamento remoto; terra firme; Amazônia.
\end{abstract}

\begin{abstract}
Biomass estimated through IKONOS II satellite of the upland Dense Ombrophilous Forest in Central Amazon. The implications of the reduction of tropical forest in the carbon cycle has created a demand for development of non destructive methods to biomass estimates a key component in modeling biogeochemical cycles. The research had as objective to estimate indirectly the above ground biomass (AGB) of a pristine forest, correlating field data to IKONOS II satellite data. Biomass was obtained by indirect method and the vegetation analysis by means of horizontal structure. The original bands, vegetation index like ARVI, NDVI, MSAVI and R43, PCA and Tasseled Cap transforms and Spectral Mixing Linear Model were used in modeling. The integration of ground truth and satellite data was performed by statistical procedure, by the application of linear multiple regression using STEPWISE method. For the spatialization, the minimum and the maximum values of the confidence interval for biomass estimates provided by the model were adopted as classes. The confidence interval was $343,05 \pm 14,90 \mathrm{Mg}$. ha ${ }^{-1}$ for $95 \%$ probability. The highest correlations between biomass and digital

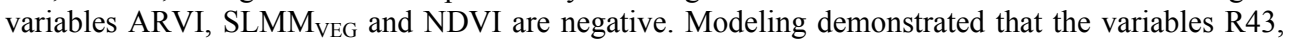
NDVI, TCap3 and TCap4 were the best correlation with biomass data.

Keywords: Above-ground biomass; modeling; remote sensing; upland forest; Amazon.
\end{abstract}

\section{INTRODUÇÃO}

As florestas tropicais vêm sendo alvo de interesse mundial, impulsionado pelas especulações sobre a redução na extensão de florestas tropicais e sua implicação no ciclo de carbono global. Segundo Skole; Tucker (1993), a conversão dessas florestas em áreas destinadas à agricultura e pastagem resulta em um fluxo contínuo de carbono para a atmosfera. Dale et al. (1993) comentam que as mudanças no uso da terra têm representado entre 30 e $50 \%$ da emissão global de $\mathrm{CO}_{2}$, colaborando para a concretização do 
chamado "efeito estufa", resultante do bloqueio da radiação de calor efetuado pelos gases, aquecendo a superfície terrestre e tendo como conseqüência as mudanças climáticas e o aumento da temperatura média global nos próximos 100 anos.

$\mathrm{Na}$ Amazônia, as florestas de terra firme situam-se, geralmente, em terrenos ondulados nas baixas altitudes (raramente excedem a $250 \mathrm{~m}$ sobre o nível do mar) e comportam no seu interior outros tipos de vegetação de pequenas comunidades florísticas, como campinas e campinaranas, representadas por ilhas, importantes para a manutenção da diversidade florística e faunística (AYRES, 1993). Segundo Tivy (1995), a variação na quantidade de biomassa também difere no número de espécies, na composição e estrutura, na abundância da população das espécies e na forma de vida da qual ela é composta.

A estimativa de biomassa florestal acima do solo das florestas tropicais úmidas da Amazônia brasileira convertendo dados dos inventários florestais foi inicialmente realizada por Brown e Lugo (1992). Todavia, essas estimativas foram realizadas a partir de dados de inventários florestais nos anos 70 , cuja finalidade era a quantificação de madeira para a exploração florestal, excluindo outras espécies, sem importância econômica (HIGUCHI et al., 2004). Outros autores, entre eles, Clark e Clark (2000), Keller et al. (2001); Houghton et al. (2001) e Castilho (2004), comentam que as estimativas de biomassa para grandes áreas devem ser obtidas a partir de amostras aleatórias, replicáveis, de tamanho adequado, dispostas de modo a cobrir toda a área em questão ou estratificadas de acordo com a classificação espacial da vegetação.

Laurance et al. (1999) quantificaram a biomassa seca acima do solo, a partir de diâmetros de 10 $\mathrm{cm}$ à altura do peito (dap $\geq 10 \mathrm{~cm}$ ) em amostras de 1 ha, totalizando 65 hectares, usando equação alométrica desenvolvida por Santos (1996) $-\mathrm{BAS}=(\exp (3,323+(2,546 \times(\ln (\mathrm{DBH} / 100)))))$ x $600-\mathrm{e}$ um fator de correção para estimar a biomassa total de árvores pequenas. As estimativas de biomassa variaram de 231 a 492 t/ha, com média de $356 \pm 47$ t/ha. Os parâmetros solo-fertilidade podem responder por 1/3 ou mais da biomassa acima do solo nas florestas de terra firme na Amazônia.

Devido à grande extensão e localização remota da região amazônica, estudos nessa região têm tido como suporte o emprego de técnicas de sensoriamento remoto, utilizando, essencialmente, imagens de sensores ópticos. As propriedades biofísicas têm sido estimadas nessa faixa do espectro eletromagnético, por meio de índices de vegetação (FOODY et al., 2003). Adicionalmente, os sistemas sensores na faixa de microondas fornecem informações sobre as propriedades estruturais dos alvos (CHIPMAN, 1997).

Estudos nas florestas da África Central com o satélite IKONOS foram realizados, correlacionando os valores espectrais e os índices obtidos das imagens com área basal e densidade de árvores para distinguir estruturas florísticas, assim como índices de diversidade e associações de espécies para definir padrões em gradientes topográficos com variações de umidade. Os resultados mostraram que as bandas espectrais e os índices de vegetação foram mais sensíveis às mudanças na composição e na estrutura florística durante a estação seca, e que o infravermelho próximo, banda 4 (MS4), foi a mais sensível em todos os gradientes topográficos (THENKABAIL et al., 2003).

Apesar da floresta Amazônica não estar contemplada pelo protocolo de Kyoto, estudos vêm demonstrando a grande importância do bioma amazônico no ciclo de carbono. Dessa forma, a necessidade de aprofundar e melhorar metodologias que possam estimar os parâmetros biofísicos de biomassa é essencial para estimar a quantidade de carbono emitida ou seqüestrada (HIGUCHI et al., 2004). Além disso, a estimativa de biomassa acima do solo é necessária para entender produtividade, alocações de nutrientes e ciclos de carbono. Com o jogo de interesses pelas cotas de captação de $\mathrm{CO}_{2}, \mathrm{o}$ sensoriamento remoto desempenha um papel primordial na quantificação das áreas e na estimativa indireta por meio dos modelos de equações desenvolvidas para cada unidade fitoecológica, fazendo-se inicialmente uso dos modelos de equação desenvolvidos para a quantificação de biomassa por meio indireto, aplicando-se os resultados desses modelos em equações desenvolvidas com os valores digitais dos sensores remotos, diminuindo os custos e tempos despendidos na quantificação clássica de biomassa.

Uma vez que a vida útil do satélite mais utilizado para estudos ambientais, o Landsat-5, encontra-se em seus dias regressivos de uso, existe a necessidade de que outros satélites sejam testados. Assim sendo, fez-se uso das imagens de alta resolução espacial do satélite IKONOS II nesta pesquisa.

No estudo aqui exposto, apresenta-se a seguinte seqüência metodológica: quantificação de biomassa na floresta ombrófila densa de terra firme pelo método indireto, processamento de imagens IKONOS II e transformações das bandas originais para a modelagem da estimativa de biomassa pelo satélite IKONOS II, em uma área da bacia do rio Puraquequara, na área central da Amazônia, em 
Manaus. Desse modo, os objetivos que nortearam toda a dimensão do estudo foram: (a) análises florísticas e estruturais das amostras da floresta ombrófila densa de terra firme; (b) quantificação da biomassa acima do solo na floresta ombrófila densa de terra firme; (c) ajuste de modelos matemáticos de biomassa florestal acima do solo com o satélite IKONOS II; e (d) espacialização da fitomassa obtida do modelo desenvolvido com os dados do satélite.

\section{MATERIAL E MÉTODOS}

\section{Descrição da área de estudo}

A área de estudo está localizada entre as coordenadas geográficas 5946’30" - 59॰54’30" Oeste e 0256’30" - 0300'00" Sul, com Datum SAD/69-Brasil. Compreende uma área aproximada de $608 \mathrm{~km}^{2}$ e está inserida na bacia do rio Puraquequara, a leste da cidade de Manaus, zona de expansão da cidade (Figura 1).

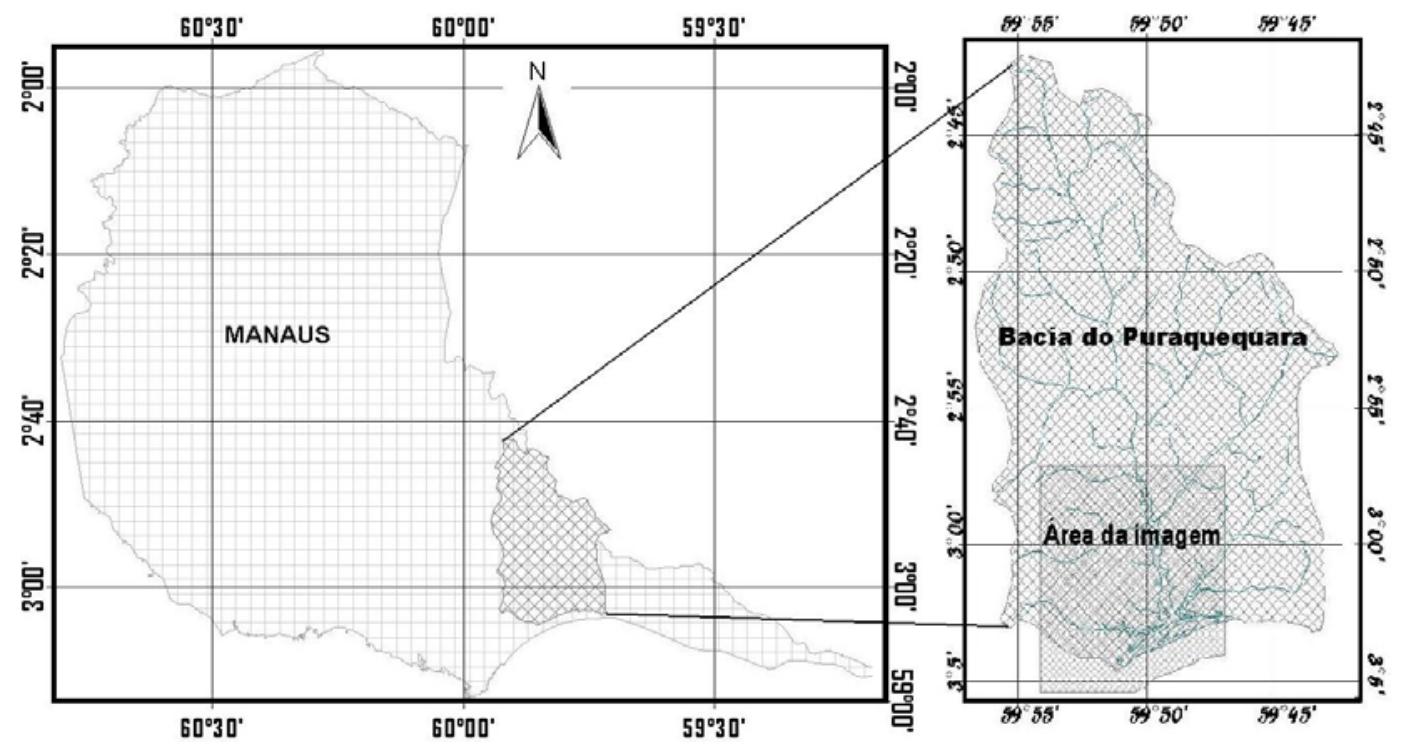

Figura 1. Área de estudo.

Figure 1. Study area.

A formação geológica presente na área é denominada Alter do Chão. Os solos dessa formação são derivados dos depósitos de sedimentos flúvios-lacustres do Terciário que foram expostos a alta temperatura e pluviosidade, resultando na remoção da maioria dos nutrientes (CHAUVEL et al., 1987; SOMBROEK, 2000; CASTILHO, 2004). O relevo se apresenta, segundo MME (1978), em sua maioria plano, tendo suas maiores altitudes em torno dos $145 \mathrm{~m}$.

Segundo o relatório do Projeto RADAMBRASIL (MME, 1978), nos levantamentos dos produtos naturais, o clima predominante na área de estudo é do tipo quente e úmido, correspondendo, na classificação de Köppen (1948), ao Am. As temperaturas médias anuais são elevadas. A média é de 26 ${ }^{\circ} \mathrm{C}$, permanecendo relativamente constante ao longo do ano (médias mensais variam cerca de $2{ }^{\circ} \mathrm{C}$ ). As chuvas concentram-se entre novembro e junho, sendo março e abril os meses de maior precipitação. A estação seca, de acordo com Marques Filho et al. (1981), concentra-se entre julho e setembro, com precipitação mensal menor que $100 \mathrm{~mm}$. Em 2003, a precipitação total anual foi de 2044,7 mm, a umidade relativa foi de $88 \%$ e a temperatura foi de $28,4{ }^{\circ} \mathrm{C}$. (estação climatológica da Reserva DUCKE/INPA).

A área de estudo é dominada em 95\% por Floresta Ombrófila Densa das Terras Baixas, de acordo com a base vetorial do banco de dados do SIPAM/origem IBGE (1992), sendo este o grande grupo fitoecológico, sem suas faciações (dossel emergente, uniforme, com ou sem palmeiras). 


\section{Material Utilizado}

A imagem IKONOS II utilizada neste estudo é do dia 25/07/2003 e foi disponibilizada pelo projeto MODIVEAM/UFPR/INPA/CNPq (Processo CNPq $n^{\circ} 479252 / 03-3$ ), possuindo as seguintes informações: ângulo de elevação solar $=58,42622$, ângulo azimutal solar $=43,7984$ e ângulo de elevação nominal de coleta $=81,144$ graus decimais.

\section{Trabalhos de campo e laboratoriais}

Os trabalhos de campo, no que diz respeito à mensuração de diâmetro à altura do peito (dap), foram realizados em dezembro/2003, julho e dezembro de 2004 e abril/maio de 2005 e constituíram-se no inventário aleatório de 24 hectares, compondo ao todo 575 amostras de $20 \times 20 \mathrm{~m}$ (Figura 2), nas quais foram determinadas as coordenadas do centro com GPS de navegação, com precisão de $8 \mathrm{~m}$ e, em suas extremidades, com GPS diferencial com precisão de $10 \mathrm{~cm}$. Os indivíduos vegetais abordados foram todos os que apresentavam dap $\geq 5 \mathrm{~cm}$, obedecendo ao critério que deu origem às fórmulas empregadas nesta pesquisa para o cálculo de biomassa fresca. O esquadrinhamento de cada hectare, composto de 25 amostras, foi desenhado em escala e georreferenciado, sobreposto nas imagens originais e transformadas, sendo obtidas as médias de cada uma das áreas de $20 \times 20 \mathrm{~m}$, composta de 25 pixels, usadas nos ajustes dos modelos.

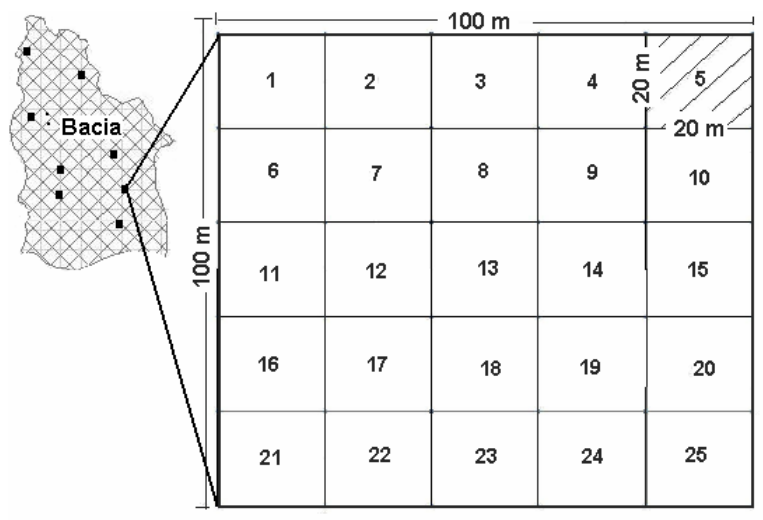

Figura 2. Localização das parcelas amostrais por hectare inventariado.

Figure 2. Surveyed-hectare plot location.

\section{Determinação da biomassa acima do solo}

Os modelos matemáticos neste trabalho, aplicados na quantificação do peso da massa fresca, foram desenvolvidos por Higuchi et al. (1994, 1998). Segundo os autores, as fórmulas apresentam resultados tão consistentes e precisos quanto os obtidos por outros modelos que se utilizam da variável altura das árvores. O desenvolvimento dos modelos aconteceram a partir de um banco de dados de 315 árvores com dap $>5 \mathrm{~cm}$, obtidos pelo método destrutivo de 5 parcelas de $400 \mathrm{~m}^{2}$, em um sítio coberto por floresta de terra firme, sobre platôs de latossolo amarelo, na Estação Experimental de Silvicultura Tropical (EEST) do INPA, aproximadamente a $90 \mathrm{~km}$ ao norte de Manaus. Esses modelos estão dispostos no tabela 1 .

Tabela 1. Modelos utilizados na quantificação de biomassa.

Table 1. Biomass quantification models.

\begin{tabular}{lccc}
\hline Critério & Equação gerada & $\mathbf{R}^{2}$ & Syx \\
\hline $1 \mathrm{a}(5<\mathrm{DAP}<20 \mathrm{~cm})$ & $\ln \mathrm{P}=-1,754+2,665 \ln (\mathrm{DAP})$ & 0,92 & 43 \\
$1 \mathrm{~b}(\mathrm{DAP}>20 \mathrm{~cm})$ & $\ln \mathrm{P}=-1,151+2,170 \ln (\mathrm{DAP})$ & 0,90 & 2035 \\
\hline
\end{tabular}

Fonte: Higuchi et al.(1994); R²: Coeficiente de determinação; Syx: erro padrão da estimativa em quilos.

A biomassa seca foi estimada a partir da subtração da concentração média de água, em madeiras da região, da biomassa úmida. O percentual de concentração de água utilizado na extração da biomassa fresca foi da ordem de 40\% para toda a árvore, como recomendado por Higuchi; Carvalho Jr. (1994), 
ficando o cálculo assim definido: $\quad \mathrm{B}_{\text {seca }}=\mathrm{B}_{\text {úmida }} \mathrm{x}(1-\mathrm{Tu})$, na qual, $\mathrm{B}_{\text {seca }}=$ biomassa seca $(\mathrm{Mg}), \mathrm{B}_{\text {úmida }}=$ biomassa úmida $(\mathrm{Mg})$ e $\mathrm{Tu}=$ teor de umidade em valor absoluto $(0,4)$.

\section{Identificação das espécies e análise da vegetação}

Durante os levantamentos dos dados de DAP, foram coletadas amostras botânicas, constituídas de galhos terminais com folhas das espécies ocorrentes nas unidades amostrais. O material botânico foi desidratado em estufa elétrica, posteriormente identificado por comparação no acervo do herbário do INPA, em alguns casos identificado por especialistas da família e classificado segundo o sistema de classificação de Cronquist (1981). Essas informações foram usadas nas análises florísticas.

A análise florística foi efetuada por sua Composição, Estrutura Horizontal, Diversidade e Similaridade. $\mathrm{Na}$ análise de diversidade, usaram-se os índices de diversidade de Shannon $\left(\mathrm{H}^{\prime}=-\sum \mathrm{p}_{\mathrm{i}}\right.$. $\ln \left(\mathrm{p}_{\mathrm{i}}\right)$, onde $\mathrm{p}_{\mathrm{i}}=\left(\mathrm{n}_{\mathrm{i}} / \mathrm{N}\right)$ é a probabilidade de um indivíduo amostrado pertencer à espécie $i ; \mathrm{n}_{\mathrm{i}}=\mathrm{n}^{\circ}$ total de indivíduos da espécie $i ; \mathrm{N}=\mathrm{n}^{\circ}$ total de indivíduos amostrados na área) e Simpson $(\mathrm{S}=1-\mathrm{D}$, onde $\mathrm{D}=$ $\sum\left(\mathrm{n}_{\mathrm{i}} / \mathrm{N}\right)^{2}$. Na análise de similaridade, foi utilizado o índice não-paramétrico (ausência e presença) de Simpson $\left(\mathrm{S} s=\mathrm{M} / \mathrm{N}_{\min }\right.$, onde $\mathrm{M}=\mathrm{n}^{\mathrm{o}}$ de espécies comuns às amostras analisadas $\mathrm{e} \mathrm{N}_{\min }=\mathrm{n}^{\mathrm{o}}$ de indivíduos da amostra com menos indivíduos), por apresentar maior peso às espécies comuns, segundo Hammer, Harper e Ryan (2005).

A análise estrutural dos dados teve como base a densidade relativa (DR), a freqüência relativa (FR), a dominância relativa (DoR) e o valor de importância das espécies (VI), calculados como recomendado por Martins (1993). Para o agrupamento das similaridades, usou-se como medida a distância euclidiana (KREBS, 1998).

\section{Pré-processamento de imagem}

A fórmula para a transformação de valores digitais em radiância foi fornecida pela Space Imaging, detentora das imagens, de acordo com o documento SE-REF-016 (FLEMING, 2003), bem como as características espectrais empregadas na transformação de radiância e expressa pela fórmula $\mathrm{L}_{\lambda}\left(\mathrm{W} \mathrm{m} \mathrm{m}^{-2}\right.$ $\left.\operatorname{sr}^{-1} \mu \mathrm{m}^{-1}\right)=(\mathrm{DN} /(\mathrm{CalCoef} / 10)) /$ Largura da banda $\lambda(\mu \mathrm{m})$, onde CalCoef $\lambda_{\lambda}=$ Coeficiente de calibração para a banda $\lambda$ e Largura da banda $\lambda=$ Largura (amplitude) da banda $\lambda$.

Os valores de radiância foram aplicados na fórmula de Markham e Barker (1986) e fazem parte do livro-texto para usuários dos dados científicos do satélite LANDSAT, usado para converter DN para valores de reflectância exoatmosférica (NASA, 2002), expressa por:

$$
\begin{aligned}
& \rho_{\rho}=\left(\pi^{*} L_{\lambda}{ }^{*} d^{2}\right) / E_{\lambda}{ }^{*} \cos \left(\theta_{s}\right) \\
& \rho_{p}=\text { reflectância planetária; } \\
& \mathrm{L}_{\lambda}=\text { radiância espectral na abertura do sensor }(\mathrm{W} \mathrm{m}-2 \mathrm{sr}-1 \mu \mathrm{m}-1) ; \\
& \text { ESUN } \mathrm{N}_{\lambda}=\text { irradiância exoatmosférica da banda }(\mathrm{W} \mathrm{m}-2 \mu \mathrm{m}-1) ; \\
& \theta_{\mathrm{s}}=\text { angulo solar zenital (convertido para radianos em processamento de imagem); } \\
& \mathrm{d}=\text { distância terra-sol (unidades astronômicas). }
\end{aligned}
$$

Na correção geométrica, fez-se uso do GPS de precisão com dupla freqüência (L2), com precisão de $10 \mathrm{~cm}$. Para a correção atmosférica, aplicou-se o modelo 6S (VERMOTE et al., 1997), adaptado por Antunes et al. (2003), tendo sido essa parte do pré-processamento realizada usando-se rotinas programadas em linguagem Legal.

\section{Transformação de imagens}

Quatro índices de vegetação e uma razão foram calculados a partir das quatro bandas individuais (MS1, MS2, MS3 e MS4), azul, verde, vermelho e infra-vermelho próximo, ficando assim formulados: 1) Índice de Vegetação Resistente à Atmosfera (ARVI) = (MS4-(2*(MS3-MS1)))/(MS4+(2*(MS3MS1))) (KAUFFMAN e TANRE, 1996); 2) Índice de Vegetação Modificado para a Interferência do Solo $(\mathrm{MSAVI})=\left(2 * \mathrm{MS} 4+1-\left((2 * \mathrm{MS} 4+1)^{2}-8 *(\mathrm{MS} 4-\mathrm{MS} 3)\right)^{1 / 2}\right) / 2(\mathrm{QI}$ et al.,1994); 3)Índice de Vegetação da Diferença Normalizada (NDVI) $=(\mathrm{MS} 4-\mathrm{MS} 3) /(\mathrm{MS} 4+\mathrm{MS} 3)$ (ROUSE et al, 1974); 4) Índice de Vegetação Ajustado para o Solo $(\mathrm{SAVI})=\left(((\mathrm{MS} 4-\mathrm{MS} 3) /(\mathrm{MS} 4+\mathrm{MS} 3+\mathrm{L}))^{*}(1+\mathrm{L})\right)(\mathrm{HUETE}, 1988)$ e valor do fator de cobertura do solo (L) =0,5; e 5) Razão43 (R43) = MS4/MS3. 
As outras transformações foram realizadas por meio de Componentes Principais, Tasseled Cap (TCap) e pelo Modelo Linear de Mistura Espectral (MLME).

Os coeficientes adotados para a geração das imagens transformadas Tasseled Cap (TCap) foram adotados de HORNE (2003), uma vez que, para o Brasil, estudos que determinem esses coeficientes ainda não foram realizados. O conjunto de equações é expresso da seguinte forma:

$$
\begin{aligned}
& \mathrm{TCap}_{1}=0,326 \times \text { blue }+0,509 \times \text { green }+0,560 \times \text { red }+0,567 \times \text { nir } \\
& \text { TCap }_{2}=-0,311 \times \text { blue }-0,356 \times \text { green }-0,325 \times \text { red }+0,819 \times \text { nir } \\
& \text { TCap }_{3}=-0,612 \times \text { blue }-0,312 \times \text { green }+0,722 \times \text { red }-0,081 \times \text { nir } \\
& \text { TCap }_{4}=-0,650 \times \text { blue }+0,719 \times \text { green }-0,243 \times \text { red }-0,031 \times \text { nir }
\end{aligned}
$$

O modelo linear de mistura espectral foi aplicado usando a técnica descrita por Keshava e Mustard (2002). A obtenção das assinaturas espectrais dos endmembers e a decomposição do pixel em imagens-fração foram estimadas pelo método de mínimos quadrados ponderados (HOLBEN; SHIMABUKURO, 1993), cuja formulação matemática é a seguinte:

$$
\begin{aligned}
& r_{i}=\sum_{j=1}^{n}\left(a_{i j} x_{j}\right)+e_{i} \quad e_{i}=r_{i}-\sum_{j=1}^{n}\left(a_{i j} x_{j}\right) \\
& r_{i}=\text { reflectância espectral observada de um pixel na banda } i, \text { contendo um ou mais componentes; } \\
& a_{i j}=\text { reflectância espectral do componente } j \text { para a banda espectral } i ; \\
& x_{j}=\text { proporção do componente } j \text { (endmember) dentro de um pixel; } \\
& e_{i}=\text { erro para a banda } i \text { (contribuição residual não considerada pelo conjunto de endmembers); } \\
& i=1,2,3, \ldots, n \text { (número de bandas espectrais envolvidas para a geração do modelo). }
\end{aligned}
$$

\section{Ajuste do modelo matemático e espacialização da estimativa de biomassa}

O procedimento empregado foi o ajuste de modelos de regressão linear múltipla, aplicando-se o método de seleção de variáveis Stepwise. O número total de variáveis utilizadas foi de 108, incluindo todas as transformações envolvidas, além das usualmente empregadas no sensoriamento, as elevadas às potências 2 e 3, bem como a raiz quadrada, o logaritmo natural e o logaritmo base 10 . O valor do pixel empregado no ajuste foi a média de mais ou menos 25 pixels (= área da parcela amostrada).

$\mathrm{O}$ modelo foi selecionado com base nos critérios $\mathrm{R}_{\text {ajustado, erro padrão da estimativa e nos níveis }}^{2}$ de significância das variáveis. O modelo escolhido foi espacializado usando-se o método de mapeamento por fatiamento. Para compor as classes de biomassa, usou-se o menor e o maior valor dos próprios dados, dos valores estimados pelo modelo, dos intervalos de confiança para a previsão e para as estimativas das médias ( $95 \%$ de confiabilidade).

De posse desse gradiente de classes, a imagem-modelo foi exportada para o ambiente do sistema de informações geográficas, para a geração do mapa de biomassa. As áreas de não-interesse foram excluídas usando-se uma máscara vetorial, criada anteriormente por interpretação visual na tela do computador, por meio dos critérios clássicos de fotointerpretação, como textura, forma, padrão e o conhecimento sobre a resposta espectral do satélite para determinados alvos, como solo, vegetação secundária, campinas, etc., mantendo-se, assim, somente as áreas de Floresta Ombrófila Densa de Terra Firme e eliminando-se todas as outras classes de cobertura e usos do solo restantes, tendo sido o resultado dessa interpretação comparado com a base vetorial de fisionomias vegetais disponibilizada pelo SIPAM/origem IBGE (1992).

\section{RESULTADOS E DISCUSSÃO}

A relação entre os parâmetros florestais e as respostas espectrais do satélite é ainda pouco entendida (LU et al., 2004) e, sabendo-se que os elementos na comunidade vegetal influenciam a resposta espectral e, portanto, afetam diretamente os modelos ajustados para a obtenção de dados biofísicos, estimados por meio de satélites óticos, o primeiro passo nesta pesquisa foi efetuar o perfil florístico das amostras que se prestaram aos modelos de regressão linear múltipla para a variável biomassa. 
O diagnóstico florístico somente das unidades que se prestaram aos ajustes dos modelos apresentou em sua composição 748 espécies, 278 gêneros e 71 famílias, totalizando 28.613 indivíduos abordados. Em estudos florísticos realizados em um hectare de área de vertente desse tipo fitoecológico a leste da área de estudo, Oliveira; Amaral (2004) encontraram 771 indivíduos/ha para dap $\geq 10 \mathrm{~cm}$. Considerando-se que os dados foram obtidos com medida de circunferência à altura do peito (CAP $=30$ $\mathrm{cm})$ e submetidos a transformação $(\mathrm{CAP} / \pi)$, alguns indivíduos estariam abaixo do critério de $10 \mathrm{~cm}$. Assim, os 650 indivíduos/ha com dap $\geq 10 \mathrm{~cm}$ deste estudo podem ser considerados com densidade similar à encontrada pelos autores.

De acordo com o valor de importância, a espécie Eschweilera coriacea (Lecythidaceae) teve maior participação na resposta espectral, floristicamente falando, tendo mantido um equilíbrio tanto em densidade quanto em dominância na área amostrada. Na tabela 2, encontram-se relacionadas 15 das 748 espécies mais importantes encontradas neste estudo.

Tabela 2. Valor de importância das 15 espécies mais importantes.

Table 2. Importance value from 15 outstanding species.

\begin{tabular}{llcccc}
\hline $\mathbf{N}^{\mathbf{0}}$ & Espécie & DR & DoR & FR & VI \\
\hline 1 & Eschweilera coriacea (DC.) S.A. Mori & 6,595 & 5,999 & 0,469 & 13,063 \\
2 & Protium apiculatum Sw. & 3,970 & 2,419 & 0,444 & 6,833 \\
3 & Arborea-Indet & 1,569 & 2,241 & 0,395 & 4,205 \\
4 & Eschweilera collina Eyma & 1,838 & 1,743 & 0,370 & 3,952 \\
5 & Licania octandra (Hoff. ex Roem \& Schult.) Kuntz. & 1,786 & 1,318 & 0,493 & 3,597 \\
6 & Corythophora alta R. Knuth & 1,118 & 1,871 & 0,493 & 3,483 \\
7 & Dicypellium manausense W.A.Rodr. & 1,611 & 1,307 & 0,419 & 3,337 \\
8 & Cipó-Indet1 & 2,422 & 0,525 & 0,370 & 3,317 \\
9 & Inga sp. & 1,632 & 1,230 & 0,370 & 3,232 \\
10 & Geissospermum sericeum Benth. \& Hook. f. ex Miers & 0,643 & 2,036 & 0,469 & 3,148 \\
11 & Micrandopsis scleroxylum (W.Rodr.) W.Rodr. & 0,919 & 1,822 & 0,370 & 3,111 \\
12 & Psidium araça Raddi & 1,786 & 0,949 & 0,370 & 3,105 \\
13 & Licania oblongifolia Standl. & 1,321 & 1,232 & 0,493 & 3,046 \\
14 & Scleronema micranthum (Ducke) Ducke & 1,003 & 1,500 & 0,493 & 2,996 \\
15 & Cariniana micrantha Ducke & 0,881 & 1,411 & 0,395 & 2,686 \\
\hline
\end{tabular}

A segunda espécie com maior VI é Protium apiculatum (Burseraceae), notadamente demonstrando que pertence ao estrato médio, cuja densidade, embora inferior à primeira, mantém a proporcionalidade com dominância, possui muitos indivíduos finos, conseqüentemente com menor dominância nas amostras, o que leva a inferir que $P$. apiculatum seria a espécie complementar a $E$. coriacea para compor a resposta espectral em muitas das amostras, dado que a área de copa está em torno de $150-250 \mathrm{~m}^{2}$ e a resposta espectral utilizada para a modelagem foi a média de mais ou menos 25 pixels $\left(400 \mathrm{~m}^{2}\right)$.

A amplitude do índice de diversidade de Shannon foi de 3,80 a 5,34, com média de 4,58 e para o índice de diversidade de Simpson a amplitude foi de 95-99\%, com média de 98\%, representando uma alta diversidade. Maciel (2002) usou o índice de Simpson em uma floresta em Moju, Pará, na mesma unidade fitoecológica, e obteve um alto percentual de diversidade, 78 a 92\%, o qual demonstra que as unidades aqui amostradas possuem diversidade superior, corroborando com o exposto por Gentry (1988), de que a Amazônia Ocidental possui diversidade superior à da Amazônia Oriental. A similaridade florística avaliada pelo índice de diversidade de Simpson está disposta na tabela 3, e a representação gráfica dos agrupamentos está disposta na figura 3.

Observando-se o dendrograma na figura 3, verifica-se que a similaridade florística média ficou em torno dos $75 \%$. Os resultados de similaridade florística obtidos neste estudo induz a concluir que, em termos de composição, a resposta espectral foi semelhante entre as áreas amostradas e que as diferenças entre os valores de pixels podem ser por conta da arquitetura das plantas e dos perfis de altura das espécies na unidade fitoecológica. 
Tabela 3. Matriz de similaridade florística de Simpson.

Table 3. Simpson's floristic similarity Matrix.

\begin{tabular}{|c|c|c|c|c|c|c|c|c|c|c|c|c|c|c|c|c|c|c|c|c|}
\hline & h2 & h3 & h4 & h5 & h6 & h7 & h8 & h10 & h11 & h12 & h13 & h14 & h15 & h16 & h18 & h19 & h20 & h22 & h23 & h24 \\
\hline h2 & 1 & & & & & & & & & & & & & & & & & & & \\
\hline h3 & 0,5 & 1 & & & & & & & & & & & & & & & & & & \\
\hline h4 & 0,5 & 0,4 & 1 & & & & & & & & & & & & & & & & & \\
\hline h5 & 0,3 & 0,4 & 0,5 & 1 & & & & & & & & & & & & & & & & \\
\hline h6 & 0,3 & 0,4 & 0,6 & 0,5 & 1 & & & & & & & & & & & & & & & \\
\hline h7 & 0,4 & 0,4 & 0,3 & 0,3 & 0,3 & 1 & & & & & & & & & & & & & & \\
\hline h8 & 0,4 & 0,3 & 0,3 & 0,3 & 0,3 & 0,7 & 1 & & & & & & & & & & & & & \\
\hline h10 & 0,4 & 0,3 & 0,3 & 0,3 & 0,3 & 0,7 & 0,8 & 1 & & & & & & & & & & & & \\
\hline h11 & 0,4 & 0,3 & 0,3 & 0,3 & 0,3 & 0,6 & 0,7 & 0,7 & 1 & & & & & & & & & & & \\
\hline h12 & 0,4 & 0,3 & 0,3 & 0,3 & 0,3 & 0,7 & 0,8 & 0,8 & 0,8 & 1 & & & & & & & & & & \\
\hline h13 & 0,4 & 0,3 & 0,3 & 0,3 & 0,3 & 0,7 & 0,8 & 0,8 & 0,7 & 0,7 & 1 & & & & & & & & & \\
\hline h14 & 0,4 & 0,3 & 0,3 & 0,3 & 0,3 & 0,7 & 0,8 & 0,8 & 0,7 & 0,8 & 0,8 & 1 & & & & & & & & \\
\hline h15 & 0,4 & 0,4 & 0,3 & 0,3 & 0,3 & 0,7 & 0,7 & 0,8 & 0,7 & 0,7 & 0,8 & 0,8 & 1 & & & & & & & \\
\hline h16 & 0,4 & 0,3 & 0,3 & 0,3 & 0,3 & 0,7 & 0,8 & 0,8 & 0,7 & 0,7 & 0,8 & 0,8 & 0,8 & 1 & & & & & & \\
\hline h18 & 0,4 & 0,3 & 0,3 & 0,3 & 0,3 & 0,7 & 0,8 & 0,8 & 0,7 & 0,8 & 0,8 & 0,8 & 0,7 & 0,8 & 1 & & & & & \\
\hline h19 & 0,4 & 0,3 & 0,3 & 0,3 & 0,3 & 0,7 & 0,8 & 0,7 & 0,7 & 0,7 & 0,8 & 0,8 & 0,8 & 0,8 & 0,7 & 1 & & & & \\
\hline h20 & 0,4 & 0,3 & 0,3 & 0,3 & 0,3 & 0,7 & 0,8 & 0,8 & 0,7 & 0,8 & 0,7 & 0,8 & 0,8 & 0,8 & 0,7 & 0,8 & 1 & & & \\
\hline h22 & 0,4 & 0,3 & 0,3 & 0,3 & 0,3 & 0,7 & 0,8 & 0,8 & 0,7 & 0,8 & 0,7 & 0,8 & 0,8 & 0,8 & 0,8 & 0,7 & 0,8 & 1 & & \\
\hline h23 & 0,4 & 0,3 & 0,3 & 0,3 & 0,2 & 0,7 & 0,7 & 0,8 & 0,7 & 0,8 & 0,7 & 0,8 & 0,7 & 0,8 & 0,8 & 0,7 & 0,7 & 0,8 & 1 & \\
\hline h24 & 0,4 & 0,3 & 0,3 & 0,3 & 0,3 & 0,7 & 0,8 & 0,8 & 0,7 & 0,8 & 0,7 & 0,7 & 0,7 & 0,8 & 0,8 & 0,7 & 0,8 & 0,8 & 0,8 & 1 \\
\hline
\end{tabular}

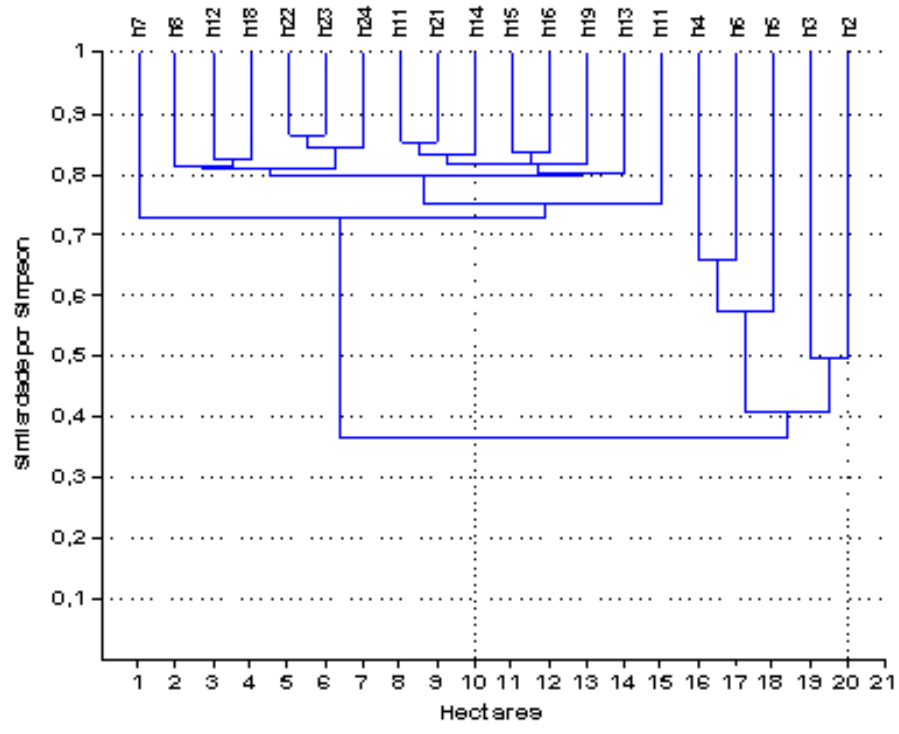

Figura 3. Dendrograma das áreas amostradas.

Figure 3. Sampled dendrogram

\section{Biomassa acima do solo na Floresta Ombrófila Densa de Terra Firme}

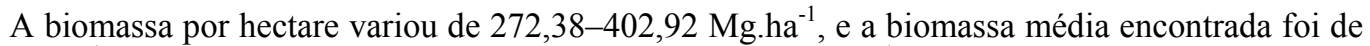
343,06 Mg.ha ${ }^{-1}(1 \mathrm{Mg}=1 \mathrm{t}=1000 \mathrm{~kg})$, com desvio de 34,45 Mg.ha-1 e intervalo de confiança (IC) de 343,05 $\pm 14,90 \mathrm{Mg} \cdot \mathrm{ha}^{-1}$. Esses resultados encontram-se dentro da amplitude obtida por Laurance et al.

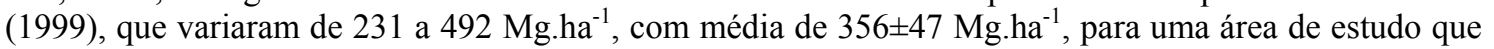
abrange a região do rio Puraquequara e outros locais da Amazônia. 


\section{Modelagem dos dados espaciais e de campo}

A matriz de correlação de Pearson entre a biomassa, bandas originais e imagens sintéticas, envolvidas nos modelos ajustados, demonstra que a correlação entre as variáveis é negativa na maior parte dos casos (Tabela 4). As correlações lineares mais fortes ocorreram com as variáveis ARVI, NDVI, MS4, MMveg (Imagem fração vegetação do modelo linear de mistura espectral). Correlações negativas de biomassa e variáveis digitais do TM em floresta primária na Amazônia Oriental foram constatadas por Maciel (2002), para a Banda $4(-0,89), M_{\mathrm{veg}}(-0,84)$ e SAVI $(-0,83)$. Lu et al. (2004), também com o TM, relatam que essa correlação é negativa em -0,538 entre biomassa acima do solo e a banda 4, para uma área de vegetação de terra firme em Altamira/Pa. Watzlawick (2003) obteve com o satélite IKONOS II correlações negativas de biomassa com as variáveis MS4, Razão, NDVI e SAVI, que variaram de 0,710,80, com melhor desempenho para MS4 (-0,80). Autores como Ardö (1992) e Trotter et al. (1997) relatam essa relação negativa entre o sensor TM do Landsat e a variável volume. Analisando-se que a banda 4 do IKONOS tem amplitude similar à do TM, era de se esperar que os parâmetros biofísicos da vegetação primária de terra firme na Amazônia também apresentassem comportamento espectral inverso às suas quantificações.

Tabela 4. Matriz de correlação de Pearson entre a variável biomassa e reflectância.

Table 4. Pearson's correlation matrix between biomass and reflectance.

\begin{tabular}{|c|c|c|c|c|c|c|c|c|c|c|c|c|c|c|}
\hline & $\begin{array}{c}\text { Biomassa } \\
(\mathbf{M g} / \mathbf{4 0 0} \\
\left.\mathbf{m}^{2}\right)\end{array}$ & MS1 & MS2 & MS3 & MS4 & ARVI & $\begin{array}{c}\text { MM } \\
\text { veg }\end{array}$ & Msavi & NDVI & R43 & PC1 & SAVI & TCap3 & RCap4 \\
\hline $\begin{array}{c}\text { Biomassa } \\
(\mathrm{Mg} / 400 \\
\left.\mathrm{m}^{2}\right)\end{array}$ & 1,000 & & & & & & & & & & & & & \\
\hline MS1 & 0,025 & 1,000 & & & & & & & & & & & & \\
\hline MS2 & $-0,034$ & $-0,230$ & 1,000 & & & & & & & & & & & \\
\hline MS3 & $-0,018$ & 0,061 & 0,211 & 1,000 & & & & & & & & & & \\
\hline MS4 & $-0,061$ & $-0,257$ & 0,536 & 0,108 & 1,000 & & & & & & & & & \\
\hline ARVI & $-0,070$ & 0,213 & 0,018 & $-0,102$ & 0,088 & 1,000 & & & & & & & & \\
\hline MMveg & $-0,061$ & $-0,278$ & 0,463 & 0,006 & 0,991 & 0,105 & 1,000 & & & & & & & \\
\hline Msavi & $-0,051$ & $-0,181$ & 0,366 & $-0,389$ & 0,848 & 0,198 & 0,895 & 1,000 & & & & & & \\
\hline NDVI & $-0,070$ & $-0,096$ & 0,339 & $-0,288$ & 0,791 & 0,275 & 0,828 & 0,934 & 1,000 & & & & & \\
\hline R43 & $-0,058$ & $-0,274$ & 0,293 & $-0,523$ & 0,703 & 0,125 & 0,761 & 0,890 & 0,766 & 1,000 & & & & \\
\hline PC1 & 0,025 & 1,000 & $-0,230$ & 0,061 & $-0,257$ & 0,213 & $-0,278$ & $-0,181$ & $-0,096$ & $-0,274$ & 1,000 & & & \\
\hline SAVI & $-0,058$ & $-0,193$ & 0,424 & $-0,222$ & 0,925 & 0,182 & 0,953 & 0,980 & 0,941 & 0,835 & $-0,193$ & 1,000 & & \\
\hline TCap3 & 0,005 & $-0,163$ & $-0,246$ & 0,758 & $-0,363$ & $-0,168$ & $-0,409$ & $-0,697$ & $-0,611$ & $-0,715$ & $-0,163$ & $-0,604$ & 1,000 & \\
\hline TCap4 & $-0,032$ & $-0,555$ & 0,833 & $-0,070$ & 0,403 & 0,020 & 0,388 & 0,397 & 0,326 & 0,394 & $-0,555$ & 0,385 & $-0,198$ & 1,000 \\
\hline
\end{tabular}

Na tabela 5, são apresentados os modelos ajustados por regressão linear múltipla. Apesar de as maiores correlações lineares de biomassa estarem com as variáveis ARVI, NDVI, MS4, MMveg, são as transformações de NDVI e MSAVI que mais estão presentes nos modelos ajustados, sendo que a combinação da variável razão R43 transformada, TCap3 e TCap4 transformadas melhor explicam a variável biomassa. Voltando aos modelos ajustados com o TM por Maciel (2002), seu modelo para todos os estratos da vegetação apresentou a banda 4 como o ajuste com o menor erro padrão da estimativa $\left(\mathrm{S}_{\mathrm{xy}}\right.$ $=23,59 \%$ ).

A escolha do modelo para espacialização baseou-se nas estatísticas usualmente empregadas ( $\mathrm{R}^{2}$, $\mathrm{R}_{\text {ajustado, }} \mathrm{S}_{\mathrm{xy}} \%, \mathrm{~F}$ ), bem como na simplicidade da replicação do modelo. A não-escolha pelo primeiro modelo foi a presença de valores estimados acima de 2,5 desvios padronizados, além da grande quantidade de transformações que seriam necessárias para a implementação do mesmo. Por sua vez, os modelos contendo as transformações TCap levam no cálculo de seus coeficientes as características dos solos americanos, cujas condições ambientais são diferentes da região amazônica, razão pela qual não foram escolhidos para serem espacializados. Assim, o resultado com essas variáveis não se apresentou tão superior aos outros modelos. Dessa forma, optou-se pela simplicidade de aplicação do modelo 
$\left(\right.$ LnBiomassa $\left.=2,5701-0,0000201951 * \mathrm{R} 43^{3}\right)$. Os resultados das avaliações estatísticas do modelo estão dispostos na tabela 6 . As avaliações de análise de resíduos estão apresentadas graficamente na figura 4.

Tabela 5. Modelos ajustados para os dados de biomassa no intervalo de $2-3,45 \mathrm{Mg} / 400 \mathrm{~m}^{2}$.

Table 5. Biomass data adjusted models in the interval of $2-13,45 \mathrm{Mg} / 400 \mathrm{~m}^{2}$.

\begin{tabular}{|c|c|c|c|c|c|c|c|}
\hline $\mathbf{N}^{\mathbf{0}}$ & Modelos COM constante & Média & D.P. & $\begin{array}{l}\mathbf{R}^{2} \\
(\%)\end{array}$ & $\begin{array}{l}R^{2} \text { aj. } \\
(\%)\end{array}$ & $S_{\mathbf{y x}}$ & $\begin{array}{l}\mathbf{S}_{\mathrm{yx}} \\
\%\end{array}$ \\
\hline 1 & $\begin{array}{c}\text { LnBiomassa }=2,45671-2,7686^{*} \mathrm{TCap}_{4}- \\
0,0138884 * \mathrm{ARVI}^{2}-0,0896523 * \mathrm{Ln}(\mathrm{NDVI}) \\
-0,0000267698^{*} \mathrm{R}^{3}-20,8903^{3} \mathrm{PC}^{3}{ }^{3} \\
50,1445^{*} \mathrm{TCap}^{3}+352,606^{*} \mathrm{TCap}^{3}\end{array}$ & 12,68 & 1,42 & 3,38 & 27,11 & 6,05 & 10,60 \\
\hline 2 & $\begin{array}{l}\text { LnBiomassa }=2,49834-1,44542 * \text { TCap4- } \\
\text { 0,0000232062*R433- } \\
\text { 21,6093*TCap33 }{ }^{3}+215,889 * \mathrm{TCap}^{3}{ }^{3}\end{array}$ & 12,68 & 1,35 & 6,05 & 2,46 & 1,40 & 10,57 \\
\hline 3 & LnBiomassa $=2,5701-0,0000201951^{*} \mathrm{R} 43^{3}$ & 12,55 & 1,42 & 3,13 & 2,51 & 1,39 & 11,08 \\
\hline 4 & $\begin{array}{l}\text { Biomassa }=12,9971+1,31441 * \operatorname{Ln}(\text { MSAVI })- \\
\quad 2,88264 * \operatorname{Ln}(\mathrm{NDVI})\end{array}$ & 13,19 & 4,22 & 0,99 & 0,54 & 4,20 & 31,87 \\
\hline 5 & 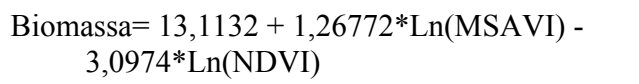 & 13,47 & 4,57 & 1,02 & 0,58 & 4,55 & 33,17 \\
\hline
\end{tabular}

Tabela 6. Modelo de regressão linear e importância da variável para o modelo LnBiomassa $=2,5701$ $0,0000201951 * \mathrm{R} 43^{3}$.

Table 6. Linear regression model and the importance of the variable for the model LnBiomassa $=$ $2,5701-0,0000201951 * \mathrm{R} 43^{3}$.

\begin{tabular}{lcccrrr}
\hline Variáveis & $\begin{array}{c}\text { Coeficientes } \\
\text { estimados }\end{array}$ & Erro padrão & Lim. Inf. & Lim. Sup. & $\boldsymbol{t}$ & $\boldsymbol{p}$ \\
\hline$\beta_{\mathrm{o}}$ & 2,5701 & 0,0167621 & 0,0167621 & 2,53715 & 153,328 & 0,0000 \\
$\mathrm{R} 43^{3}$ & $-0,0000201951$ & 0,0000889851 & 0,0000088985 & $-0,0000376832$ & $-2,26949$ & 0,0237 \\
\hline \multicolumn{7}{c}{ Análise de Variância do modelo de biomassa para o IKONOS II } \\
\hline Fonte de variação & S.Q. & G.L. & M.Q. & F & $p$ \\
\hline Modelo & 0,5574 & 1 & 0,557415 & $5,15^{* *}$ & 0,0237 \\
Resíduo & 48,3759 & 447 & 0,108223 & & \\
Total & 48,9333 & 448 & & & \\
**P $\left(\mathrm{F}_{\mathrm{c}}>\mathrm{F}_{t}\right): 95 \% \mathrm{~F}_{(447,1)}: 1,96$ & & &
\end{tabular}

Fazendo um paralelo, os resultados de ajuste de biomassa obtidos por Watzlawick (2003) para a Floresta Ombrófila Mista com o IKONOS II possuem uma aproximação de similaridade estrutural arquitetônica com a Floresta Ombrófila Densa, resguardadas as diferenças ambientais entre ambas, a equação obtida nesse estudo teve melhor desempenho no ajuste, $\mathrm{R}_{\text {ajustado }}=2,51 \%$ e $\mathrm{S}_{\mathrm{xy}}=11,08 \%$ contra $\mathrm{R}^{2}{ }_{\text {ajustado }}=0,73 \%$ e $\mathrm{S}_{\mathrm{xy}}=22,59 \%$. Talvez isso se reflita em razão da floresta amazônica, na região de Manaus, ter em seu estrato abaixo do dominante muitos indivíduos de menor porte que coíbem a reflectância do solo, aumentando a resposta espectral da vegetação, uma vez que o pixel menor implica maior resolução espacial, conseqüentemente as plantas menores passam a ter papel importante na composição do valor de reflectância do pixel. Os casos de florestas com maior número de indivíduos arbóreos grandes passam a representar um problema, devido à passagem da reflectância do solo e a grande diferença na arquitetura do dossel.

Em estudos realizados no âmbito do experimento em larga escala da biosfera e atmosfera na Amazônia (LBA, ASNER e WARNER, 2003), sobre a variação da fração de sombra nas coberturas florestais ao longo das florestas amazônicas brasileiras, os autores concluíram que a média global de sombras dentro dos talhões florestais foi de $0,25 \pm 0,12 \%$. Essa distribuição de sombra foi tida como assimétrica, com $30 \%$ dos pixels tendo valores fracionais de sombra acima de 0,30. Fatos como este, 
agregados à baixa resolução espectral do IKONOS, são o que tornam seus ajustes tão inferiores, se comparados aos do Landsat.

Em trabalho realizado no Sul de Camarões, África, área contida na zona de floresta tropical úmida, com satélites de banda larga, entre eles o IKONOS II, para modelar e quantificar a biomassa de árvores, arbustos e outros, Thenkabail (2004) concluiu que os sensores comparados possuem séria limitação na modelagem de biomassa, conseguindo explicar somente $13-60 \%$ da variabilidade da biomassa das florestas primárias, e observou que os melhores resultados foram obtidos com o satélite Hyperion (hiperespectral), que explicou a variabilidade da biomassa de $36-83 \%$ mais que os outros satélites (IKONOS II, ETM+, ALI).
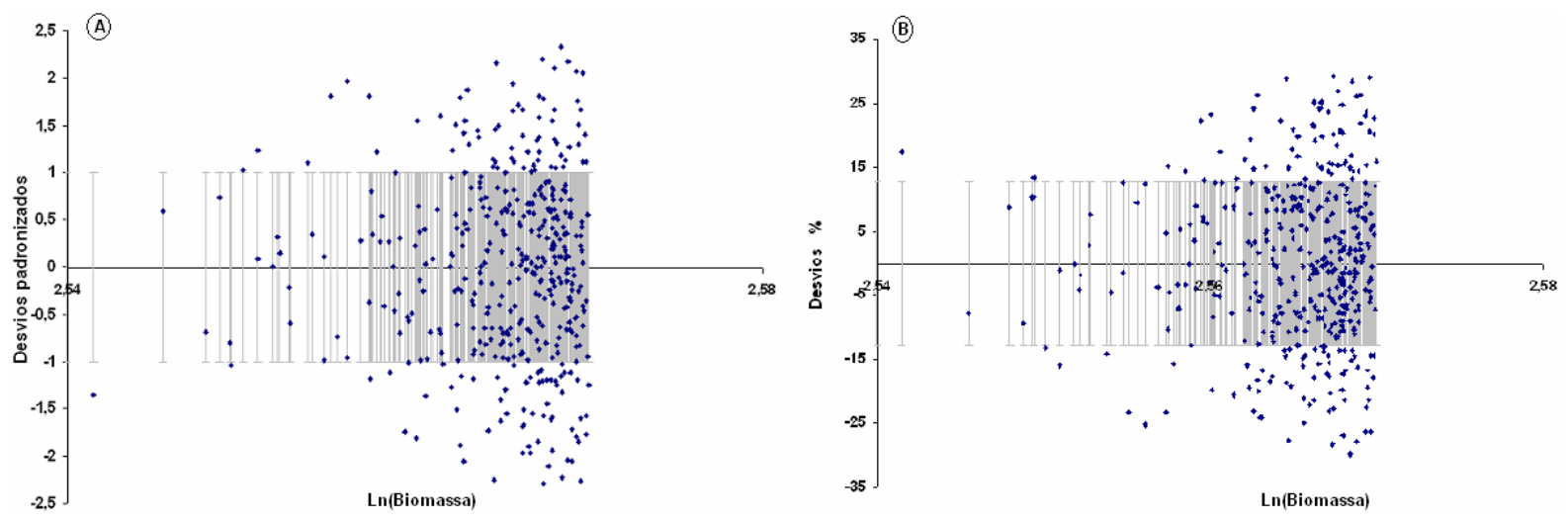

Figura 4. Análise gráfica dos resíduos do modelo linear para a biomassa. em $400 \mathrm{~m}^{2}$. a) resíduos padronizados; b) resíduos percentuais.

Figure 4. Linear model residual graphical analysis for biomass in $400 \mathrm{~m}^{2}$. a) standardized residuals; b) percentile residuals.

\section{Espacialização de biomassa para o satélite IKONOS II}

A espacialização dos dados de biomassa para o modelo IKONOS II (LnBiomassa $=2,5701$ $\left.0,0000201951 * \mathrm{R} 43^{3}\right)$ é apresentada na figura 5 - Bacia do Puraquequara. Os critérios da espacialização encontram-se dispostos na tabela 7.

Tabela 7. Critérios e classes para a espacialização da biomassa em $400 \mathrm{~m}^{2}$.

Table 7. Criteria and classes for biomass spatiality in $400 \mathrm{~m}^{2}$.

\begin{tabular}{lr}
\hline Critérios & Classes (Ln(Biomassa)) \\
\hline MeVaLi dos valores estimados para o desvio padrão ATÉ o MeVaLi dos valores & $1,60-2,05$ \\
estimados para a média & $2,05-2,29$ \\
MeVaLi dos valores estimados para a média ATÉ o MeVa dos dados estimados & $2,29-2,55$ \\
MeVa dos dados estimados ATÉ a média dos dados ajustados & $2,55-2,57$ \\
\hline
\end{tabular}

MeVa: Menor valor; Li: Limite inferior; exemplo: MeVaLi: Menor valor do limite inferior

\section{CONCLUSÕES}

As espécies E. coriacea e $P$. apiculatum preencheram os requisitos de maiores valores de freqüência, dominância e densidade e, conseqüentemente, estruturalmente foram as mais importantes e que mais contribuíram para a resposta espectral.

Os resultados da modelagem mostraram que as variáveis R43, NDVI, TCap 3 e TCap 4 foram as que melhor se correlacionaram com a biomassa da Floresta Ombrófila Densa de Terra Firme. 


\section{BACIA DO PURAQUEQUARA - LN (BIOMASSA)}

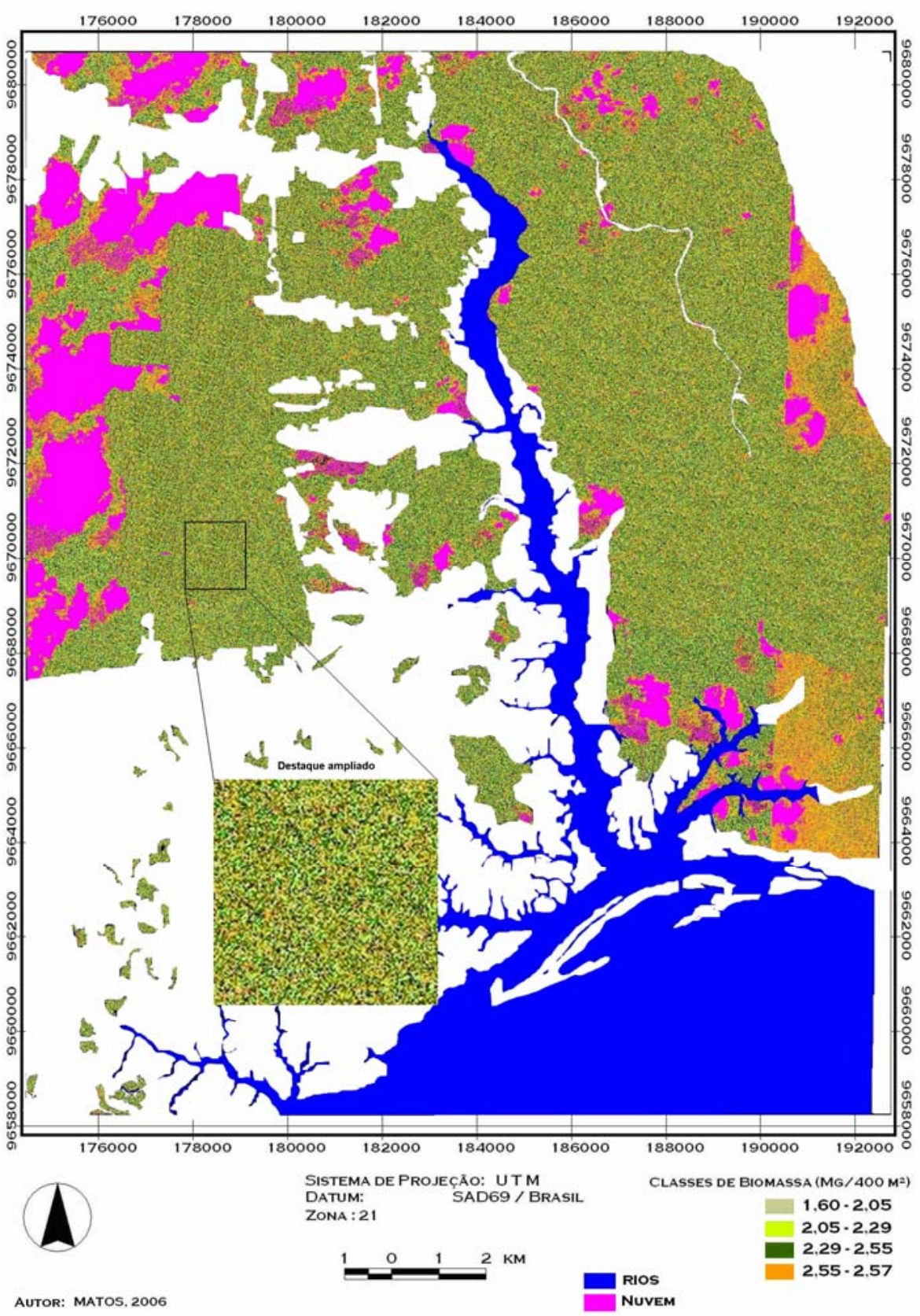

Figura 5. Imagem resultante da modelagem de biomassa.

Figure 5. Image resulting from biomass modeling.

\section{AGRADECIMENTOS}

Ao Conselho Nacional de Desenvolvimento Científico e Tecnológico (CNPq), pelo apoio financeiro de bolsa de estudo e do Projeto MODIVEAM (Monitoramento Espacial da Dinâmica da Vegetação de Várzea e Terra Firme no Estado do Amazonas), Processo No. 479252/2003-3, e à Coordenação de Pesquisas em Botânica/INPA, pelo apoio logístico. 


\section{REFERÊNCIAS}

ANTUNES, M. A. H.; FREIRE, R. M. B.; BOTELHO, A. S.; TONIOLLI, L. H. Correções Atmosféricas de Imagens de Satélites utilizando o Modelo 6S. In: XXI CONGRESSO BRASILEIRO DE CARTOGRAFIA, 21., Belo Horizonte Anais do... Rio de Janeiro: Sociedade Brasileira de Cartografia, 2003.

ARDÖ, J. Volume quantification of coniferous forest compartments using spectral radiance recorded by Landsat Thematic Mapper. International Journal of Remote Sensing, Basingstoke, v. 13, n. 9, p. 17791786, 1992.

ASNER, G. P.; WARNER, A. S. Canopy shadow in IKONOS satellite observations of tropical forests and savannas. Remote Sensing of Environment, New York, v. 87, p. 521-533, 2003.

AYRES, J. M., As Matas de Várzea do Mamirauá. [Brasília, DF]: MCT-CNPq/Programa do Trópico Úmido - Sociedade Civil Mamirauá. 1993. 123 p.

BRASIL. MINITÉRIO DAS MINAS E ENERGIA. Departamento. Nacional de Produção Mineral. Projeto RADAMBRASIL: Folha AS-20, Purus. Brasília, 1978. v. 18.

BROWN, S.; LUGO, A. E. Aboveground biomass estimates for tropical moist forests of the Brazilian Amazon. Interciencia, Caracas, v. 17, n. 1, p. 8-18, 1992.

CASTILHO, C. V. de. Variação espacial e temporal da biomassa arbórea viva em $64 \mathbf{~ k m}^{2}$ de floresta de terra-firme na Amazônia Central. 72 p Tese (Doutorado em Ciências Biológicas) - Instituto Nacional de Pesquisas da Amazônia, Universidade Federal do Amazonas, Manaus, 2004.

CHAUVEL, A.; LUCAS, Y.; BOULET, R. On the genesis of the soil mantle of the region of Manaus, Central Amazonia, Brazil. Experientia, [S.1.], v. 43, p.234-241, 1987.

CHIPMAN, J. W. Comparison of TM, ERS-1, and SIR-C Data for forest type mapping in the Lake States. In: ACSM/ASPRS - Annual Convention \& Exposition, Seattle, Washington, Technical Papers. Bethesda: ASPRS, 1997. v. 3, p. 517-526.

CLARCK, D. B.; CLARCK, D. A. Landscape-scale variation in forest structure and biomass in a tropical rain forest. Forest Ecology and Management, Amsterdan, v. 137, n. 1-3, p. 185-198, 2000.

CRONQUIST, A. An integrated system of classification flowering plants. New York: Columbia University Press, $1981.126 \mathrm{p}$.

DALE, V. H.; PEDLOWSKI, M. A.; O’NEILL, R. V. O. Southworth, F. Alternativas para a Amazônia. Ciência Hoje, Sâo Paulo, v. 16, n. 993, p. 61-64, ago. 1993.

FLEMING, D. Convertion from DN to planetary refelectance. Version 3.0. Nov. 2003. Revisão de: Convertion from DN to Planetary Reflectance, Space Imaging Document Number SE-REF-016, Rev. A, 3 p. 2003. Disponível em <http;//www.sapceimaging.com/productos/ikonos/spectral.html $>$. Acesso em: 26/02/2005.

FOODY, G. M.; BOYD, D. S.; CUTLER, M. E. J. Predictive relations of tropical Forest biomass from Landsat TM data and their transferability between regions. Remote Sensing of Environment, New York, v. 85 , p. 463-474. 2003.

GENTRY, A. H. Changes in plant community diversity and floristic composition on environmental and geographical gradients. Annals of the Missouri Botanical Garden, Saint Louis, MO, v. 75, p. 1-34, 1988.

HAMMER, Ø.; HARPER, D. A. T.; RYAN, P. D. PAST - PAlaeontological Statistics, ver. 1.37. disponível em < http://folk.uio.no/chammer/past>. Acesso em: 22/06/2005.

HIGUCHI, N.; CARVALHO JR., J. A. Fitomassa e conteúdo de carbono de espécies arbóreas da Amazônia, in: SEMINÁRIO EMISSÃO X SEQÜESTRO DE CO2: UMA NOVA OPORTUNIDADE DE 
NEGÓCIOS PARA O BRASIL, 1994, Rio de Janeiro. Anais do... Rio de Janeiro: Companhia Vale do Rio Doce, 1994. p. 125-153.

HIGUCHI, N.; SANTOS, J. M. dos; IMANAGA, M.; YOSHIDA, S. Aboveground biomass estimate for Amazonian dense tropical moist forests. Memoirs of the Faculty of Agriculture, Kagoshima, JP, v. 30, n. 39, p. 43-54, 1994.

HIGUCHI, N.; SANTOS, J. dos; RIBEIRO, R. J.; MINETTE, L.; BIOT,Y. Biomassa da parte aérea da vegetação da floresta tropical úmida de terra-firme da Amazônia brasileira. Acta Amazônica, Manaus,.v. 28, n. 2, p. 153-166, 1998.

HIGUCHI, N.; CHAMBERS, J.; SANTOS, J. dos; RIBEIRO, R. J.; PINTO, A. C. M.; SILVA, R. P. da; ROCHA, R. M.; TRIBUZY, E. S. Dinâmica e balanço do carbono da vegetação primária da Amazônia Central. Manaus, Floresta, Curitiba, v. 34, n. 3, p. 295-304, set./dez. 2004.

HOLBEN, B. N.; SHIMABUKURO, Y. E. Linear mixing model applied to AVHRR Lac data. In: SIMPÓSIO BRASILEIRO DE SENSORIAMENTO REMOTO, 7., 1993, Curitiba,. Anais... São José dos Campos: INPE, 1993. v. 2, p. 102-110.

HORNE, J. H. A tasseled cap transformation for ikonos images. ASPRS 2003 Annual Conference Proceedings. Anchorage, Alaska, USA, May, 2003. 9 p.

HUETE, A. R. A Soil-Adjusted Vegetation Index (SAVI). Remote Sensing of Environment, New York, v. 25, n. 3, p. 295-309, 1988.

HOUGHTON, R. A.; LAWRENCE, K. T.; HACKLER, J. L.; BROWN, S. The spatial distribution of forest biomass in the Brazilian Amazon: a comparison of estimates. Global Change Biology, Osford, GB, v. 7, p. 731-746, 2001.

INSTITUTO BRASILEIRO DE GEOGRAFIA E ESTATÍSTICA (IBGE). Departamento de Recursos Naturais e Estudos Ambientais. Manual técnico da vegetação brasileira. Rio de Janeiro, 1992. 92 p. (Manuais Técnicos em Geociências, 1).

KAUFMAN, Y. J.; TANRE, D. Strategy for direct and indirect methods for correcting the aerosol effect on remote sensing: From AVHRR to EOS-MODIS. Remote Sensing of Environment, New York, n. 55, p. 65-79, 1996.

KELLER, M.; PALACE, M.; HURTT, G. Biomass estimation in the Tapajós National Forest, Brazil. Examination of sampling and allometric uncertainties. Forest Ecology and Management, Amsterdan, v. 154, n. 3, p. 371-382, 2001.

KESHAVA, N.; MUSTARD, J. F. Spectral Unmixed. IEEE Signal Processing Magazine, New York, v. 19, n. 1, p. 44-57, 2002.

KÖPPEN, W. Climatologia. México: Fondo de Cultura Económica, 1948.

KREBS, C. J. Ecological methodology. 2 ed. Menlo Park: Benjamin/Cummings, 1999. 620 p.

LAURANCE, W. F.; FEARNSIDE, P. M.; LAURANCE, S. G.; DELAMONICA, P.; LOVEJOY, T. E.; RANKIN-DE MERONA, J. M.; CHAMBERS, J. Q.; GASCON, C. Relationship between soils and Amazon forest biomass: a landscape-scale study. Forest Ecology and Management, Amsterdan, v. 118, n. 1-3, p. 127-138, 1999.

LU, D.; MAUSEL, P.; BRONDIZIO, E; MORAN, E. Relationship between forest stand parameters and Landsat TM spectral responses in the Brazilian Amazon Basin. Forest Ecology and management, Amsterdan, v. 198, n. 1-3, p. 149-167, Jul., 2004.

MACIEL, M. N. M. Estimativa de parâmetros estruturais de uma floresta primária na Amazônia Oriental através de dados orbitais. 144 f. Tese (Doutorado em Ciências Florestais) - Setor de Ciências Agrárias, Universidade Federal do Paraná, Curitiba, 2002. 
MARKHAM, B. L.; BARKER, J. L. Landsat MSS ant TM pos-calibration dynamics ranges, exoatmospheric reflectances and at-Satellite temperatures. EOSAT Landsat Technical Notes, [S.1.], 21 p., Aug., 1986.

MARQUES FILHO, A. O.; RIBEIRO, M. N. G.; SANTOS, J. M. Estudos climatológicos da Reserva Florestal Ducke, Manaus, AM. IV - Precipitação. Acta Amazonica, Manuas, v.4, p.759-768, 1981.

MARTINS, F. R. Estrutura de uma Floresta Mesófila. Campinas: UNICAMP, 1993. 295 p.

MATOS, F. D. A. Estimativa de biomassa e carbono em Floresta Ombrófila Densa de Terra Firme na Amazônia Central, Manaus - Am, Brasil, por meio de dados de satélites de média e alta resolução espacial. 146 f. Tese (Doutorado em Engenharia Florestal) - Setor de Ciências Agrárias, Universidade Federal do Paraná, Curitiba, 2006.

NASA. 2002. Disponível em: <http://ltpwww.zulu.ssc.gov/MrSid>. Acesso em:10/08/2003.

QI, J.; CHEHBOUNI, A.; HUETE, A. R.; KERR, Y. H.; SOROOSHIAN, S. A modified soil adjusted vegetation index. Remote Sensing of Environment. New York, v. 47, p. 1-25. 1994.

ROUSE, J. W.; HAAS, R. W.; SCHELL, J. A.; DEERING, D. W.; HARLAN, J. C. Monitoring the vernal advancement and retrogradation (Greenwave effect) of natural vegetation. NASA/GSFCT Type III Final report, Greenbelt, MD, USA. 1974.

SANTOS, J. Análise de modelos de regressão para estimar a fitomassa da floresta tropical úmida de terra-firme da Amazônia Brasileira. 175 f. Tese (Doutorado em Ciências Florestais) - Curso de Ciência Florestal, Universidade Federal de Viçosa, MG, 1996.

SKOLE, D.; TUCKER, C. Tropical deforestation and habitat fragmentation in the Amazon: Satellite data from 1978 to 1988, Science, United States, v. 260, p.1905-1910, 1993.

SOMBROEK, W. G. Amazon land forms and soils in relation to biological diversity. Acta Amazonica, Manaus, v. 30, p. 81-100, 2000.

THENKABAIL, P. S.; ENClONA, E. A.; ASHTON, M. S.; LEGG. C.; DIEU, M. J. D. Hyperion, IKONOS, ALI, and ETM+ sensors in the study of African rainforests. Remote Sensing of Environment. New York, v. 90, p. 23-43, 2004.

THENKABAIL, P. S.; HALL, J.; LIN, T.; ASHTON, M. S.; HARRIS, D.; ENCLONA, E. A. Detecting floristic structure and pattern across topographic and moisture gradients in a mixed species Central African forest using IKONOS and Landsat-7 ETM+ images International Journal of Applied Earth Observation and Geoinformation, Enschede, v. 4, p. 255-270, 2003.

TIVY, J. Biogeography : a study of plants in the ecosphere. 3.ed. England: Longman Group UK, 1995. $452 \mathrm{p}$.

TROTTER, C. M.; DYMOND, J. R.; GOULDING, C. J. Estimation of volume timber in a coniferous plantation forest using Landsat TM. International Journal of Remote Sensing, Basingstoke, v. 18, n. 10, p. 2209-2223, 1997.

VERMOTE, E. F.; TANRÉ, D.; DEUZÉ, J. L.; HERMAN, M.; MORCRETE, J. J. Second Simulation of the Satellite Signal in the Solar Spectrum, 6S: An overview. IEEE Transactions on Geoscience and Remote Sensing. New York, v. 35, n. 3, p. 675-686, 1997.

WATZLAWICK, L. F. Estimativa de biomassa e carbono em Floresta Ombrófila Mista e plantações florestais a partir de dados de imagens do satélite IKONOS II. 119 f. Tese (Doutorado em Ciências Florestais) - Setor de Ciências Agrárias, Universidade Federal do Paraná, Curitiba, 2003. 